\title{
Signal enhancement using a robust adaptive total least squares algorithm
}

\author{
Jun’ya Shimizu, Yoshikazu Miyanaga, and Koji Tochinai \\ Division of Electronics and Information Engineering, Graduate School of Engineering, \\ Hokkaido University, \\ N13 W8, Kita-ku, Sapporo, 060 Japan
}

(Received 31 January 1996)

\begin{abstract}
An adaptive enhancement algorithm of signal disturbed by the impulse noise and the white Gaussian noise is proposed in this paper. If the strong dependence between signal enhancement and needed-parameter estimation exists in an algorithm, it is difficult to reduce a large bias occurring in the first estimation. To weaken their relationship, it is considered to introduce a total least squares (TLS) algorithm which estimates model parameters directly from disturbed signals. However, the TLS estimation accuracy is dramatically deteriorated by the impulse noise, even if the TLS estimation accuracy is held to the non-Gaussian noise in some degree. Hence, we ensure the robustness of the algorithm by replacing a high amplitude signal with an estimated value based on a likelihood ratio test. Using these signals, we develop an algorithm based on the TLS and the EM algorithm for enhancing the disturbed signal. We also show the effectiveness of the proposed algorithm through computer simulations.
\end{abstract}

Keywords : Signal enhancement, Kalman filtering, Robust estimation, Total least squares algorithm, EM algorithm

PACS number: 43. 50. Ki, 43. 60. Pt, 43. 72. Ew

\section{INTRODUCTION}

Acoustical signal processing under noisy environments has often been needed. Hence it is necessary to enhance low signal to noise ratio (SNR) signals in many acoustical application fields. ${ }^{3,4,10-12,14,15)}$ In addition, an adaptive enhancement method is preferable since real-time noise reduction is needed in many cases.

For sinusoids or nearly periodic signals in the presence of white noise, it has been reported that enhancement algorithms using comb filters achieve 3-10 dB enhancement., ${ }^{40)}$ On the other hand, many algorithms without assuming the periodic signal need multi-sensor measurement, ${ }^{11,14)}$ frequency-domain processing, ${ }^{3,15)}$ and a large computational burden such as inverse matrix calculation. ${ }^{11)}$ In addition, it has often been assumed that the observation noise is the white Gaussian noise, not the non-Gaussian noise. In real environments of signal measurement, the white Gaussian noise as well as the impulse noise such as a click or a human error exists. However, an adaptive time-domain signal enhancement algorithm with a small computational burden under the non-Gaussian noise has not been reported.

In Ref. 11), a multi-sensor gradient-based algorithm has been also proposed under environments that the white Gaussian noise exists. We can reform this multi-sensor algorithm as a single-sensor algorithm with slight modification: the algorithm iterates the following procedures. First, the noisy signal is enhanced by the Kalman filtering. Secondly, model parameters, an input power level, and a noise power level, which are used in the Kalman filtering, are estimated using enhanced signals. However, these iterative signal enhancement and parameter estimation procedures are strongly depen- 
dent on each other. Hence, if a large bias occurs in the Kalman filtering or in parameter estimation, it is difficult to reduce the bias in a few iteration. To weaken the relationship between the signal enhancement procedure and the parameter estimation procedure, it is necessary to introduce a procedure which directly estimates signal parameters from observation signals, not from enhanced signals. Since we model the desired signal as an autoregressive (AR) process, we need to estimate AR parameters, the input power level, and the noise power level as signal parameters. Among preceding parameters, we estimate AR parameters directly from disturbed signals, as we shall show the algorithm in subsequent development.

One of direct estimation algorithms of AR parameters from disturbed signals is a total least squares (TLS) algorithm, which has been introduced in a numerical analysis literature. ${ }^{5)}$ Hence we use the TLS algorithm for AR parameter estimation. However, we consider the non-Gaussian noise environment that the impulse noise as well as the white Gaussian noise exists. Although the TLS estimation accuracy is held to the non-Gaussian noise in some degree, the TLS estimation accuracy is dramatically deteriorated by the impulse noise. ${ }^{9)}$ Hence, it is necessary to develop a robust adaptive signal enhancement algorithm under environments containing the impulse noise and the white Gaussian noise. Assuming the foregoing, a robust adaptive signal enhancement algorithm with a single-sensor is proposed.

\section{PROBLEM FORMULATION}

The basic problem of interest is illustrated in Fig. 1. We assume that a microphone measures the sum of the desired signal $s(t)$ and the noise $v(t)$ : the microphone output $z(t)$ is

$$
z(t)=s(t)+v(t)
$$

where

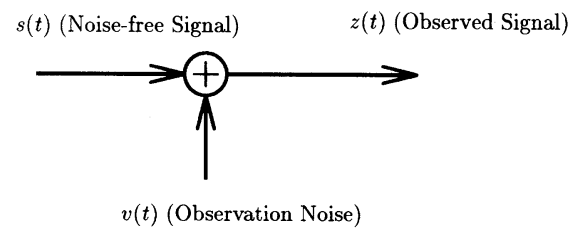

Fig. 1 Signal enhancement problem.

$$
\begin{aligned}
s(t) & =-\sum_{k=1}^{p} \alpha_{k} s(t-k)+u(t) \\
& =-\left[\begin{array}{lll}
\alpha_{1} & \cdots & \alpha_{p}
\end{array}\right]\left[\begin{array}{c}
s(t-1) \\
\vdots \\
s(t-p)
\end{array}\right]+\dot{u}(t) \\
& \triangleq-\boldsymbol{\alpha}^{\mathrm{T}} \boldsymbol{s}(t)+u(t)
\end{aligned}
$$

where $\alpha_{k}(k=1, \cdots, p)$ is an autoregressive (AR) parameter, $u(t)$ is a zero mean white Gaussian process with an average power of $\sigma_{u}^{2}$, and $v(t)$ is the zero mean white Gaussian process with the average power of $\sigma_{v}^{2}$ with a probability $1-q$ or an impulse value with the probability $q(0<q \ll 1)$. The signals $u(t)$ and $v(t)$ are mutually independent.

The objective is to generate $\hat{s}(t)$ based on measurements of $z(t)$ in such a way that a mean square error (MSE) is minimized. The minimum mean square error (MMSE) estimate $\hat{s}(t)$ is given by the conditional expectation using signal observations up to time $t$ :

$$
\widehat{s}(t)=E\{s(t) \mid z(1), z(2), \cdots, z(t)\}
$$

where $E\{\cdot \mid \cdot\}$ denotes a conditional expectation operator.

Toward this end, we represent Eqs. (1) and (2) in a state-space form as

$$
\begin{gathered}
\boldsymbol{x}(t)=\boldsymbol{\Phi} \boldsymbol{x}(t-1)+\boldsymbol{e}_{1} u(t) \\
z(t)=\boldsymbol{e}_{1}{ }^{\mathrm{T}} \boldsymbol{x}(t)+v(t)
\end{gathered}
$$

where $\boldsymbol{x}(t)$ is the $(p+1) \times 1$ state vector defined by

$$
\boldsymbol{x}(t)=\left[s(t)_{s}(t-1) \cdots s(t-p)\right]^{\mathrm{T}}
$$

$\boldsymbol{\Phi}$ is the $(p+1) \times(p+1)$ transition matrix

$$
\boldsymbol{\Phi}=\left[\begin{array}{ccccc}
-\alpha_{1} & -\alpha_{2} & \cdots & -\alpha_{p} & 0 \\
1 & 0 & \cdots & \cdots & 0 \\
0 & \ddots & & & \vdots \\
\vdots & \ddots & \ddots & & \vdots \\
0 & \cdots & 0 & 1 & 0
\end{array}\right]
$$

and $\boldsymbol{e}_{1}$ is the $(p+1) \times 1$ unit vector.

$$
\boldsymbol{e}_{1}=[10 \cdots 0]^{\mathrm{T}}
$$

If we assume that the parameters $\alpha, \sigma_{u}{ }^{2}$, and $\sigma_{v}{ }^{2}$ are precisely known and $v(t)$ is the white Gaussian process with the probability 1 , then we can compute the conditional expectation Eq. (3) recursively using the Kalman filtering equations. The first element of $\hat{\boldsymbol{x}}(t \mid t)$ is the MMSE estimate $\hat{s}(t)$. 


$$
\begin{aligned}
& \widehat{\boldsymbol{x}}(t \mid t-1)=\boldsymbol{\Phi} \widehat{\boldsymbol{x}}(t-1 \mid t-1) \\
& \boldsymbol{P}(t \mid t-1)=\boldsymbol{\Phi} \boldsymbol{P}(t-1 \mid t-1) \boldsymbol{\Phi}^{\mathrm{T}}+\sigma_{u}{ }^{2} \boldsymbol{e}_{1} \boldsymbol{e}_{1}^{\mathrm{T}} \\
& \widehat{\boldsymbol{x}}(t \mid t)=\hat{\boldsymbol{x}}(t \mid t-1) \\
& +\boldsymbol{k}(t)\left[z(t)-\boldsymbol{e}_{1}^{\mathrm{T}} \hat{\boldsymbol{x}}(t \mid t-1)\right] \\
& \boldsymbol{P}(t \mid t)=\left[\boldsymbol{I}-\boldsymbol{k}(t) \boldsymbol{e}_{1}^{\mathrm{T}}\right] \boldsymbol{P}(t \mid t-1)
\end{aligned}
$$

where $\hat{\boldsymbol{x}}(t \mid t)$ denotes an estimated state vector and $\hat{\boldsymbol{x}}(t+1 \mid t)$ denotes a prediction state vector based on data up to time $t . \quad \boldsymbol{P}(t \mid t)$ and $\boldsymbol{P}(t+1 \mid t)$ denote their error covariance matrices, respectively. $I$ denotes an identity matrix and $\boldsymbol{k}(t)$ denotes the Kalman gain given by

$$
\boldsymbol{k}(t)=\frac{1}{\boldsymbol{e}_{1}^{\mathrm{T}} \boldsymbol{P}(t \mid t-1) \boldsymbol{e}_{1}+\sigma_{v}{ }^{2}} \boldsymbol{P}(t \mid t-1) \boldsymbol{e}_{1}
$$

However, we assume that $v(t)$ has a high amplitude value with the probability $q$. Conventional methods for processing such impulse noise are put into two categories. One computes optimal estimates assuming the mixture probability distribution of $v(t){ }^{1)}$ The other method considers the data containing the impulse noise as the incomplete observation data. ${ }^{2,7)}$ In this paper, we ensure the robustness of the algorithm by replacing the high amplitude signal with an estimated value to enhance the signal using the Kalman filtering. With this point in mind, we develop a parameter estimation algorithm in the next section.

\section{SIGNAL PARAMETER ESTIMATION}

The Kalman filtering, equations require $\boldsymbol{\alpha}, \sigma_{u}{ }^{2}$, and $\sigma_{v}{ }^{2}$. Since these parameters are not available, they must be estimated as well. First, we put the observed signal to a likelihood ratio test. If we get judgement that the observed signal contains the impulse noise from the test, we replace the observed signal with the estimated signal. For this end, we introduce an index $\varepsilon(t)$ which indicates that the additive noise is the white Gaussian noise or the impulse noise :

$$
\varepsilon(t)= \begin{cases}0, & \text { white Gaussian noise } \\ 1, & \text { impulse noise }\end{cases}
$$

The likelihood ratio test algorithm will be described later.

Using these signals from which impulse noises are eliminated, we perform the maximum likelihood
(ML) estimation of signal parameters. Denote by $\boldsymbol{\theta}$ the vector of signal parameters :

$$
\boldsymbol{\theta}=\left[\boldsymbol{\alpha}^{\mathrm{T}}, \sigma_{u}{ }^{2}, \sigma_{v}{ }^{2}\right]^{\mathrm{T}}
$$

The best estimate of $\boldsymbol{\theta}$ would be to compute the ML estimate of $\boldsymbol{\theta}$ :

$$
\boldsymbol{\theta}_{\mathrm{ML}}=\arg \max _{\boldsymbol{\theta}} \log f_{Z}(\boldsymbol{z} ; \boldsymbol{\theta})
$$

where $z$ is the $t \times 1$ vector denoted by $[z(1), \cdots$, $z(t)]^{\mathrm{T}}$ and $\log f_{z}(\boldsymbol{z} ; \boldsymbol{\theta})$ is the log-likelihood of the observed signal $z$.

\subsection{AR Parameter Estimation}

In this subsection, we develop an algorithm for obtaining the ML estimate of $\boldsymbol{\alpha}$. We estimate $\boldsymbol{\alpha}$ using observed signals since we want to weaken the dependency between the signal enhancement procedure and the parameter estimation procedure. If a large bias occurs in the first estimation, the bias is reduced in a few iteration for this AR parameter estimation step.

In the focusing problem, all obtained signals are affected by noise. In addition, since we consider the case that $\sigma_{u}{ }^{2}$ is less than $\sigma_{v}^{2}$, we can consider the focusing AR model as an errors-in-variables (EIV) model. The conventional least squares (LS) algo-

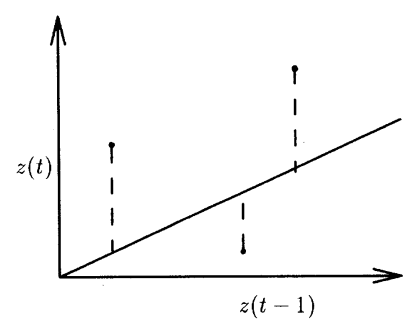

(a) LS estimation

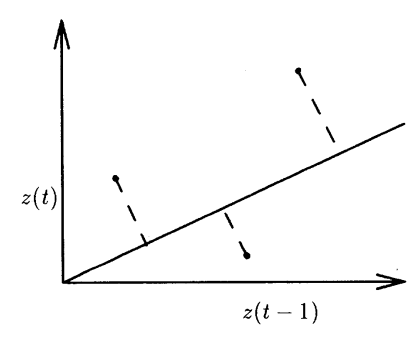

(b) TLS estimation

Fig. 2 Geometric interpretations of estimation algorithms (1-order AR model). (a) LS estimation. (b) TLS estimation. 
rithm assumes that the observation noises are free in the past signals $\{z(t-1), \cdots, z(t-p)\}$. Hence, the LS estimate results from minimizing the sum of squared vertical distances from each observation data point $[z(t) z(t-1) \cdots z(t-p)]^{\mathrm{T}}$ to a hyperplane spanned by $\boldsymbol{\alpha}$ and a vector $\boldsymbol{h}(t)=[z(t-1) \cdots$ $z(t-p)]^{\mathrm{T}}$ based on a linear prediction theory (Fig. 2(a)). However, this assumption is not suitable for this EIV model. The ML estimate of the EIV model which takes all observation noises into consideration results from minimizing the sum of squared perpendicular distances from each observation data point to the hyperplane. ${ }^{6)}$ This geometric interpretation of the focusing problem coincides with that of a total least squares (TLS) algorithm ${ }^{5}$ ) (Fig. 2(b)). Hence, the ML estimate $\alpha_{\mathrm{ML}}$ is obtained from the TLS algorithm.

From the preceding geometric interpretation, the TLS solution with AR parameters is given by minimizing the following function:

$$
E_{\mathrm{tls}}=E\left\{\frac{\left(-\boldsymbol{\alpha}^{\mathrm{T}} \boldsymbol{h}(t)-z(t)\right)^{2}}{\|\boldsymbol{\alpha}\|^{2}+1}\right\}
$$

where $\|\cdot\|$ denotes the vector norm.

To minimize adaptively the preceding cost function, an anti-Hebbian learning algorithm is proposed in Ref. 13), in which a gradient vector as a parameter-updated direction is derived from linearizing the differentiation of the instantaneous cost function. We use this algorithm with slight modification for AR parameter estimation:

$$
\boldsymbol{\alpha}(t+1)=\boldsymbol{\alpha}(t)-\mu e(t)[-\boldsymbol{h}(t)+\boldsymbol{\alpha}(t) z(t)]
$$

Here we have defined:

$$
\begin{gathered}
\widehat{z}(t)=-\boldsymbol{\alpha}^{\mathrm{T}}(t) \boldsymbol{h}(t) \\
e(t)=\bar{z}(t)-z(t)
\end{gathered}
$$

A step gain $\mu$ is asymptotically decreased to a small value or is kept at a small value. As seen from the preceding equation, this algorithm has the small computational burden.

3.2 Estimation of Input and Noise Power Levels

Next, we develop an adaptive algorithm to obtain the ML estimates of $\sigma_{u}{ }^{2}$ and $\sigma_{v}{ }^{2}$ using $\boldsymbol{\alpha}_{\mathrm{ML}}$ obtained from the TLS algorithm. It is difficult to maximize directly $\log f_{Z}(\boldsymbol{z} ; \boldsymbol{\theta})$ concerning with $\sigma_{u}{ }^{2}$ and $\sigma_{v}{ }^{2}$. Hence we maximize indirectly $\log f_{Z}(\boldsymbol{z} ; \boldsymbol{\theta})$ based on an expectation-maximization (EM) algorithm.
We begin by denoting the complete data to be $\boldsymbol{y}=$ $\left[\boldsymbol{z}^{\mathrm{T}}, \boldsymbol{s}^{\mathrm{T}}\right]^{\mathrm{T}}$, where $\boldsymbol{s}=[s(1) \cdots s(t)]^{\mathrm{T}}$. Consequently, the EM algorithm for the problem is defined as 2):

$$
\begin{aligned}
& E \text { step: Compute } U(\boldsymbol{\theta}, \widehat{\boldsymbol{\theta}}(t)) \\
& M \text { step: } \max _{\boldsymbol{\theta}} U(\boldsymbol{\theta}, \widehat{\boldsymbol{\theta}}(t)) \rightarrow \widehat{\boldsymbol{\theta}}(t+1)
\end{aligned}
$$

where

$$
U(\boldsymbol{\theta}, \widehat{\boldsymbol{\theta}}(t))=E\left\{\log f_{Y}(\boldsymbol{y} ; \boldsymbol{\theta}) \mid \boldsymbol{z} ; \widehat{\boldsymbol{\theta}}(t)\right\}
$$

and $\log f_{Y}(\boldsymbol{y} ; \boldsymbol{\theta})$ denotes the log-likelihood of $\boldsymbol{y}$. The vector $\hat{\boldsymbol{\theta}}(t)$ denotes the estimate of $\boldsymbol{\theta}$.

Under the ergodic condition, $U(\boldsymbol{\theta}, \hat{\boldsymbol{\theta}}(t))$ is obtained as follows :

$$
\begin{aligned}
U(\boldsymbol{\theta}, \widehat{\boldsymbol{\theta}}(t))= & E\left\{\log f_{S}(\boldsymbol{s} ; \boldsymbol{\theta})\right. \\
& \left.+\log f_{Z \mid S}(\boldsymbol{z} \mid \boldsymbol{s} ; \boldsymbol{\theta}) \mid \boldsymbol{z} ; \widehat{\boldsymbol{\theta}}(t)\right\}
\end{aligned}
$$

with

$$
\begin{aligned}
f_{S}(\boldsymbol{s} ; \boldsymbol{\theta})= & \prod_{k=1}^{t} \frac{1}{\sqrt{2 \pi \sigma_{u}{ }^{2}}} \\
& \cdot \exp \left\{-\frac{\left(s(k)+\boldsymbol{\alpha}^{\mathrm{T}} \boldsymbol{x}_{\phi-1}(k-1)\right)^{2}}{2 \sigma_{u}{ }^{2}}\right\}
\end{aligned}
$$

and

$$
\begin{aligned}
f_{Z \mid S}(\boldsymbol{z} \mid \boldsymbol{s} ; \boldsymbol{\theta})= & \prod_{k(\varepsilon=0)} \frac{1}{\sqrt{2 \pi \sigma_{v}{ }^{2}}} \\
& \cdot \exp \left\{-\frac{(z(k)-s(k))^{2}}{2 \sigma_{v}{ }^{2}}\right\}
\end{aligned}
$$

where $\boldsymbol{x}_{p-1}=[s(t-1) \cdots s(t-p)]^{\mathrm{T}}$.

Then

$$
\begin{aligned}
& U(\boldsymbol{\theta}, \hat{\boldsymbol{\theta}}(t))=C-\frac{t}{2} \log \sigma_{u}{ }^{2}-\frac{1}{2 \sigma_{u}{ }^{2}} \sum_{k=1}^{t}\left[\left\langle s^{2}(k)\right\rangle\right. \\
& +2 \alpha^{\mathrm{T}}\left\langle s(k) \boldsymbol{x}_{p-1}(k-1)\right\rangle \\
& \left.+\boldsymbol{\alpha}^{\mathrm{T}}\left\langle\boldsymbol{x}_{p-1}(k-1) \boldsymbol{x}_{p-1}{ }^{\mathrm{T}}(k-1)\right\rangle \boldsymbol{\alpha}\right] \\
& -\frac{N_{(\varepsilon=0)}}{2} \log \sigma_{v}{ }^{2}-\frac{1}{2 \sigma_{v}{ }^{2}} \sum_{k(\varepsilon=0)}\left[z^{2}(k)\right. \\
& \left.-2 z(k)\langle s(k)\rangle+\left\langle s^{2}(k)\right\rangle\right]
\end{aligned}
$$

where $C$ is a constant which is independent of $\sigma_{u}{ }^{2}$ and $\sigma_{v}{ }^{2} . \quad N_{(\varepsilon=0)}$ denotes a sample number judged as $\varepsilon(k)=0$. In addition, $\langle\cdot\rangle$ denotes $E\{\cdot \mid \boldsymbol{z} ; \widehat{\boldsymbol{\theta}}(t)\}$. This completes derivation of the E step.

To derive the structure of the $\mathrm{M}$ step, we maximize $U(\boldsymbol{\theta}, \hat{\boldsymbol{\theta}}(t))$ as given by Eq. (21). Since Eq. (21) expresses $U(\boldsymbol{\theta}, \widehat{\boldsymbol{\theta}}(t))$ as the sum of two parts, one of which depends only on $\sigma_{u}{ }^{2}$ and the other of which depends only on $\sigma_{v}^{2}$, the maximization can be broken down into two independent parts. Hence, we 


\section{J. SHIMIZU et al. : SIGNAL ENHANCEMENT USING ROBUST TLS ALGORITHM}

get the following ML estimate equations :

$$
\begin{aligned}
& \widehat{\sigma_{u}^{2}}(t+1) \\
& =\frac{1}{t}\left[\sum_{k=1}^{t}\left\langle s^{2}(k)\right\rangle+2 \boldsymbol{\alpha}^{\mathrm{T}} \sum_{k=1}^{t}\left\langle s(k) \boldsymbol{x}_{p-1}(k-1)\right\rangle\right. \\
& \left.+\boldsymbol{\alpha}^{\mathrm{T}} \sum_{k=1}^{t}\left\langle\boldsymbol{x}_{p-1}(k-1) \boldsymbol{x}_{p-1}{ }^{\mathrm{T}}(k-1)\right\rangle \boldsymbol{\alpha}\right] \\
& \widehat{\sigma_{u}^{2}}(t+1)=\frac{1}{N_{(\varepsilon=0)}} \sum_{k(\varepsilon=0)}\left[z^{2}(k)-2 z(k)\langle s(k)\rangle\right. \\
& \left.\quad+\left\langle s^{2}(k)\right\rangle\right]
\end{aligned}
$$

Although the estimates $\left\langle s(k) x_{p-1}(k-1)\right\rangle$, $\left\langle s^{2}(k)\right\rangle,\left\langle\boldsymbol{x}_{p-1}(k-1) \boldsymbol{x}_{p-1}{ }^{\mathrm{T}}(k-1)\right\rangle$ are needed in Eqs. (22) and (23), they are computed using $\hat{x}(t \mid t)$ and $\boldsymbol{P}(t \mid t)$ estimated from the Kalman filtering equations as follows :

$$
\begin{aligned}
\sum_{k=1}^{t}\left\langle\boldsymbol{x}(k) \boldsymbol{x}^{\mathrm{T}}(k)\right\rangle & =\left[\begin{array}{ll}
x_{11}(t) & \boldsymbol{x}_{12}(t) \\
\boldsymbol{x}_{21}(t) & \boldsymbol{X}_{22}(t)
\end{array}\right]=\boldsymbol{X}(t) \\
& =\left\langle\boldsymbol{x}(t) \boldsymbol{x}^{\mathrm{T}}(t)\right\rangle+\boldsymbol{X}(t-1) \\
\left\langle\boldsymbol{x}(t) \boldsymbol{x}^{\mathrm{T}}(t)\right\rangle & =\hat{\boldsymbol{x}}(t \mid t) \hat{\boldsymbol{x}}^{\mathrm{T}}(t \mid t)+\boldsymbol{P}(t \mid t)
\end{aligned}
$$

Hence Eqs. (22) and (23) are rewritten as the following recursive algorithms :

$$
\begin{gathered}
\widehat{\sigma_{u}^{2}}(t+1)=\frac{1}{t}\left[x_{11}(t)+\boldsymbol{x}_{12}(t) \boldsymbol{\alpha}\right] \\
\widehat{\sigma_{v}^{2}}(t+1)= \\
\frac{t-1}{t} \widehat{\sigma_{v}^{2}}(t)+\frac{\varepsilon(t)}{t} \widehat{\sigma_{v}^{2}}(t) \\
+\frac{1-\varepsilon(t)}{t}\left[z^{2}(t)-2 z(t) \hat{s}(t \mid t)\right. \\
\left.+\left\langle s^{2}(t)\right\rangle\right]
\end{gathered}
$$

where we have used the equation, $\boldsymbol{\alpha}=-\boldsymbol{X}_{22}{ }^{-1}(t)$ $\cdot \boldsymbol{x}_{21}(t)$, to derive Eq. (26).

\subsection{Likelihood Ratio Test}

Finally, we develop a likelihood ratio test algorithm for impulse noise detection. Based on the observed signals $\boldsymbol{z}$ and estimated parameters $\hat{\boldsymbol{\theta}}$, the conditional expectation of the probability of $\varepsilon=$ $[\varepsilon(1), \cdots, \varepsilon(t)]^{\mathrm{T}}$ is proportional to the following equation :

$$
\begin{aligned}
& \prod_{k=1}^{t} f(\langle v(k)\rangle) \operatorname{Pr}(\varepsilon(k)) \\
& \quad=\prod_{k=1}^{t} \exp \{-\langle h(k, \varepsilon(k))\rangle / 2\} \operatorname{Pr}(\varepsilon(k))
\end{aligned}
$$

where $f(v(k))$ is a probability density function of $v(k)$. $\operatorname{Pr}(\varepsilon(k))$ is the probability that $\varepsilon(k)$ becomes 0 or 1 , and $h(k, \varepsilon(k))=(1-\varepsilon(k))\left(\log \sigma_{v}{ }^{2}\right.$ $\left.+[z(k)-s(k)]^{2} / \sigma_{v}^{2}\right)+\varepsilon(k)\left(\log \sigma_{v}{ }^{2}+1\right)$.

Hence we can perform the following likelihood ratio test :

$$
\begin{aligned}
& \text { If } \\
& \quad \begin{array}{l}
\exp \left\{\left(1-\left[z^{2}(t)-2 z(t) \widehat{s}(t \mid t-1)\right.\right.\right. \\
\left.\left.\left.\quad+\left\langle s^{2}(t \mid t-1)\right\rangle\right] / \widehat{\sigma_{v}^{2}}(t)\right) / 2\right\} \operatorname{Pr}(\varepsilon=0) \geq \operatorname{Pr}(\varepsilon=1)
\end{array}
\end{aligned}
$$

then $\varepsilon(t)=0$, otherwise $\varepsilon(t)=1$.

Using this likelihood ratio test, we judge whether the observed signal has the statistically expected amplitude or not. If the observed signal does not contain the impulse noise, it leads a value of the exponential function in the left-hand side of Eq. (29) to about 1. Hence, if the test of Eq. (29) is satisfied, the test gives judgement that the observed signal does not contain the impulse noise. Accordingly, if the test of Eq. (29) is not satisfied, the test gives judgement that the observed signal contains the impulse noise. When we get judgement that $\varepsilon(t)$ is 1 from the preceding test, we replace $z(t)$ with the estimate $\hat{z}(t)$ as given by Eq. (15).

\subsection{Proposed Algorithm}

Hence the proposed algorithm is summarized as follows :

1. Do the likelihood ratio test using Eq. (29).

(a) If we get the result that $\varepsilon(t)=1$, then we replace the observed signal with the estimated value obtained from Eq. (15).

2. The enhancement signal is computed from the Kalman filtering equations (Eqs. (6)(10)) using $\alpha(t), \sigma_{u}^{2}(t)$, and $\sigma_{v}^{2}(t)$.

3. The AR parameters are computed using the gradient adaptive TLS algorithm Eq. (14).

4. Input and noise power levels are estimated (Eqs. (26)-(27)) using the estimated statevector in Step. 2 and AR parameters in Step. 3.

5. $t \leftarrow t+1$ and go back to the Step. 1 .

To initialize the Kalman filtering equations, we have set $\boldsymbol{P}(0 \mid 0)=c_{1} \boldsymbol{I}$, where $c_{1}$ is an arbitrarily positive constant. We have also initially set $\widehat{\sigma_{v}}{ }^{2}(1)$ $=c_{2}$ and $\widehat{\sigma_{v}}(1)=c_{3}$, where $c_{2}$ and $c_{3}$ are arbitrarily positive constants. We have initially set other quantities to be zero for the simplicity.

If we may consider that the speed of statistical property evolution in the observed signal is slow, we can alter the time-average operation $1 / t$ in the preceding algorithm to $(1-\lambda) /\left(1-\lambda^{t}\right)$ using a forgetting factor $\lambda(<1)$ to get the tracking capability.

The computational complexity of the proposed algorithm is $O\left(p^{2}\right)$ per iteration. In view of the 
sparse structure of the matrix $\boldsymbol{\Phi}$ and the vector $\boldsymbol{e}_{1}$, many multiplications and additions in the Kalman filtering are eliminated in practice. This will be also beneficial to implementation of the proposed algorithm. On the other hand, the computational complexity of conventional adaptive methods in the time-domain, such as the method using inverse matrix calculation ${ }^{11)}$ or the method using singular value decomposition, ${ }^{9)}$ is $O\left(p^{3}\right)$ per iteration. In addition, the multi-sensor enhancement algorithm ${ }^{11)}$ has greater computational cost than the proposed algorithm since the multi-sensor algorithm needs to estimate more parameters.

\subsection{Discussion}

In derivation of the preceding algorithm, the desired signal is modeled as the Gaussian AR process and the noise is modeled as the mixture of the white Gaussian process and the impulse signal. Since the algorithm does not assume the specific distribution of impulse noise, it is robust to both the sparse impulse noise and the burst impulse noise. We derive the enhancement algorithm based on the preceding modeling, and shall evaluate its performance to noise through computer simulations in the next section. The reason for such modeling of noise in simulations are given below : in real environments of signal measurement, the additive observation noise generated by multiple sources is often modeled as the white Gaussian process. For example, when the noise of cooling fan in some computers are measured at the same time, this noise is modeled as the white Gaussian noise. In addition, the shock noise generated by opening and shutting a door or some collisions is modeled as the impulse noise. Impulse noises caused by mechanical reason in measurement or transmission, such as the click noise or the burst noise, also exist. Hence, we have adopted the mixture of the white Gaussian process and the impulse signal as the model of noise.

Conventional single-sensor enhancement algorithms need to detect intervals containing only noise and to estimate the noise power level from intervals. However, the proposed algorithm does not need such detection since it can perform under environments that both desired signals and noise exist. This point also leads the proposed algorithm to various actual applications.

However, if the proposed algorithm is used under environments that both desired signals and colored noises whose spectral shapes are unknown exist together, the proposed algorithm may have a degraded performance since the proposed algorithm does not assume such environments.

\section{COMPUTER SIMULATIONS}

Computer simulations have been carried out to demonstrate the performance of the proposed algorithm using a narrow band synthesized signal and a real speech signal. The narrow band signal has been generated from the zero-mean white Gaussian process with the average power 10.0 through the 337 taps FIR filter, which has a passband $[0.05,0.07]$ on a normalized frequency. The real speech has been obtained from the speech database of $\mathrm{ATR}^{8)}$ with gain normalization. To these signals, we have added the noises which consist of the mixture of the white Gaussian process and the binomial distribution process. These noise-added signals have been used as observation signals in simulations (the SNR has been about $0 \mathrm{~dB}$ ). We have also compared the proposed algorithm with the gradient-based algorithm in Ref. 11).

\subsection{Narrow Band Signal}

Conditions of the simulation have been set as follows :

- The AR model order has been 4 .

- The appearance probability of impulse noise has been $2 \%$.

- The step size $\mu$ has linearly been decreased from 0.1 to 0.001 during first 1,000 sample points. In other sample points, $\mu$ has been kept at 0.001 .

Figure 3(a) shows the observed waveform $(1,000-$ points data segments are shown). Figure 3(b) shows the noise-free waveform. Figure 3(c) shows the enhanced waveform by the proposed algorithm. Figure 3(d) shows the enhanced waveform by the algorithm in Ref. 11). The result in Fig. 3(d) still contains the impulse noise as well as the white Gaussian noise. This shows that the adaptive algorithm in Ref. 11) needs many iteration steps to reduce noises and has no robustness to the impulse noise. However, the result of the proposed algorithm removes additive noises effectively and $7.6 \mathrm{~dB}$ enhancement has been achieved.

\subsection{Real Speech Signal}

Conditions of the simulation have been set as 


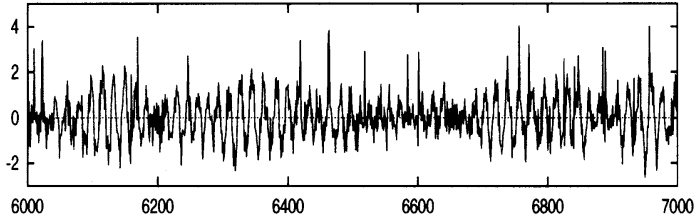

(a) Observed signal.

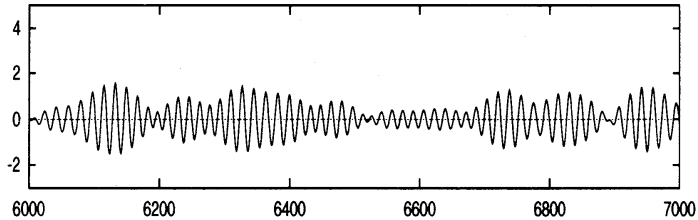

(b) Noise-free signal.

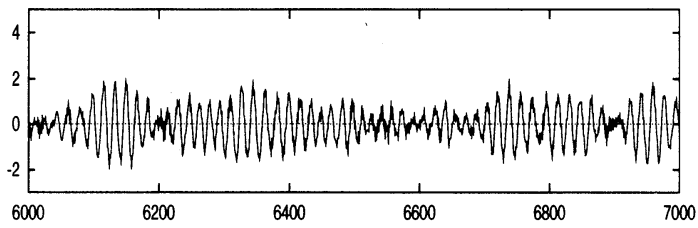

(c) Enhanced signal using the proposed algorithm.

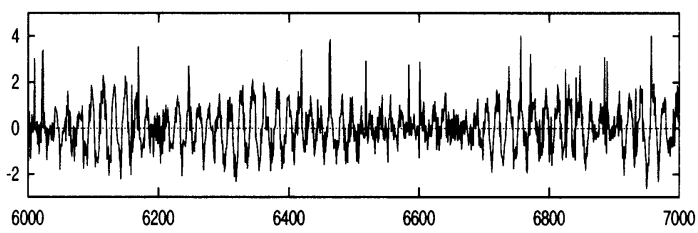

(d) Enhanced signal using the algorithm in 11).

Fig. 3 Simulation results using a narrow band signal. (a) Observed signal. (b) Noise-free signal. (c) Enhanced signal using the proposed algorithm. (d) Enhanced signal using the algorithm in Ref. 11).

follows :

- The AR model order has been 4.

- The appearance probability of impulse noise has been $0.5 \%$.

- The step size $\mu$ has linearly been decreased from 0.1 to 0.04 during first 1,000 sample points. In other sample points, $\mu$ has been kept at 0.04 .

- $\lambda=0.96$

Figure 4(a) shows the observed speech $(10,000-$ points data segments are shown). Figure 4(b) shows the noise-free waveform. Figure 4(c) shows the enhanced waveform by the proposed algorithm. Figure 4(d) shows the enhanced waveform by the algorithm in Ref. 11). The result in Fig. 4(d) still contains the impulse noise as well as the white Gaussian noise. On the other hand, the result of the proposed algorithm removes additive noises effectively and $6.3 \mathrm{~dB}$ enhancement has been

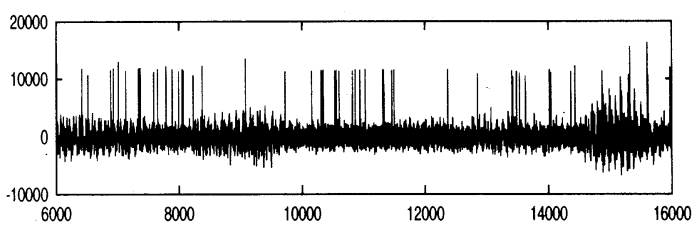

(a) Observed signal.
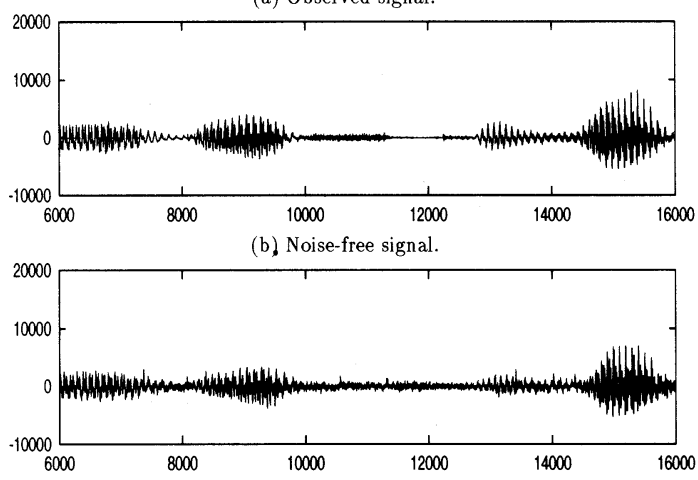

(c) Enhanced signal using the proposed algorithm.

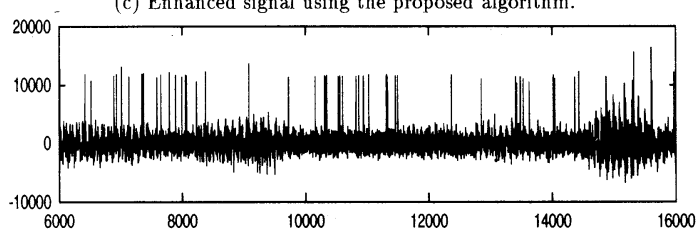

(d) Enhanced signal using the algorithm in 11).

Fig. 4 Simulation results using a real speech. (a) Observed signal. (b) Noise-free signal. (c) Enhanced signal using the proposed algorithm. (d) Enhanced signal using the algorithm in Ref. 11).

achieved. The articulation of enhanced signal has also been improved. However, the preceding enhancement value contains the following effect. First, the gradient-based TLS algorithm for estimating AR parameters in the proposed algorithm leads to the slow tracking capability in transient periods of speech. Secondly, the AR modeling in the Kalman filtering may not be appropriate to consonants, particularly fricatives. Indeed, results in stationary periods of speech have shown the higher enhancement than $6.3 \mathrm{~dB}$.

\section{CONCLUSIONS}

In this paper, we have proposed the adaptive algorithm for enhancing signal under environments that impulse and white Gaussian noises exist. First, we have described the state space model of the focusing problem for using the Kalman filtering. Secondly, we have developed the robust adaptive 
algorithm for estimating parameters needed in the Kalman filtering. In this development, first we have introduced the likelihood ratio test to get the robustness : if we get judgement that the observation signal contains the impulse noise, we replace the signal with the estimated value and we estimate the signal parameter using these signals which do not contain the impulse noise. Next, we have introduced the TLS algorithm for estimating AR parameters. This step has a role for weakening the relationship between the signal enhancement procedure and the parameter estimation procedure. Hence the initial bias is reduced in a few iteration. Using the estimated state vector in the Kalman filtering and the TLS-estimated AR parameters, we have developed the ML estimation algorithm of input and noise power levels. Iterating these procedures, we get the enhanced signal based on the MSE criterion. We have also confirmed the effectiveness of the proposed algorithm through computer simulations.

We have used the gradient-based TLS algorithm for AR parameter estimation. However, it is necessary to develop a low computational cost algorithm with high tracking capability. It is also important to evaluate the stability of the estimated AR parameters. Furthermore, it is necessary to evaluate the relationship between the noise power and the enhancement accuracy. Based on this evaluation, we should consider improvement of the algorithm to the low-power desired signal such as the fricative. These evaluations will be issues for future analysis.

\section{ACKNOWLEDGEMENT}

The authors would like to specially thank Prof. Nobuhiro Miki of Graduate School of Engineering at Hokkaido University for his useful discussion. This study was supported in part by a Grant-in-Aid for JSPS Fellows (No. 0984) and by a Grant-in-Aid for Scientific Research (No. 06808032) administered by The Ministry of Education, Science, Sports and Culture of Japan.

\section{REFERENCES}

1) C. J. Masreliez and R. D. Martin, "Robust Bayesian estimation for linear model and robustifying the Kalman filter," IEEE Trans. Autom. Control AC-22, 361-371 (1977)

2) A. P. Dempster, N. M. Laird, and D. B. Rubin, "Maximum likelihood from incomplete data via the EM algorithm,” J. R. Stat. Soc. B 39, 1-38 (1977).
3) J. S. Lim and A. V. Oppenheim, "All-pole modeling of degraded speech," IEEE Trans. Acoust. Speech Signal Process. ASSP-26, 197-210 (1978).

4) J. S. Lim, A. V. Oppenheim, and L. D. Braida, "Evaluation of an adaptive comb filtering method for enhancing speech degraded by white noise addition," IEEE Trans. Acoust. Speech Signal Process. ASSP26, 354-358 (1978).

5) G. H. Golub and C. F. Van Loan, "An analysis of the total least squares problem," SIAM J. Numer. Anal. 17, 883-893 (1980).

6) K. V. Fernando and H. Nicholson, "Identification of linear systems with input and output noise: the Koopman-Levin method," IEE Proc. D 132, 30-36 (1985).

7) M. Tanaka and T. Katayama, "A robust identification of a linear system with observation outliers by the EM algorithm," Trans. Inst. Syst. Control Inf. Eng. 1, 117-126 (1988) (in Japanese).

8) M. Abe, Y. Sagisaka, T. Umeda, and H. Kuwabara, "Speech database user's manual (continuous speech data)," ATR Tech. Rep. TR-I-166 (1990) (in Japanese)

9) S. V. Huffel and J. Vandewalle, The Total Least Squares Problem: Computational Aspects and Analysis (SIAM, Philadelphia, 1991), p. 248.

10) M. Feder, "Parameter estimation and extraction of helicopter signals observed with a wide-band interference," IEEE Trans. Signal Process. 41, 232-244 (1993).

11) E. Weinstein, A. V. Oppenheim, M. Feder, and J. R. Buck, "Iterative and sequential algorithms for multisensor signal enhancement," IEEE Trans. Signal Process. 42, 846-859 (1994).

12) H. Kobatake, "Restoration of speech waveform by maximum likelihood estimation," J. Acoust. Soc. Jpn. (J) 50, 809-815 (1994) (in Japanese).

13) K. Gao, M. O. Ahmad, and M. N. S. Swamy, "A constrained anti-Hebbian learning algorithm for total least-squares estimation with application to adaptive FIR and IIR filtering," IEEE Trans. Circuits Syst. II 41, 718-729 (1994).

14) S. Nandkumar and J. H. L. Hansen, "Dual-channel iterative speech enhancement with constraints on an auditory-based spectrum," IEEE Trans. Speech Audio Process. 3, 22-34 (1995).

15) Y. Ephraim and H. L. Van Trees, "A signal subspace approach for speech enhancement," IEEE Trans. Speech Audio Process. 3, 251-266 (1995).

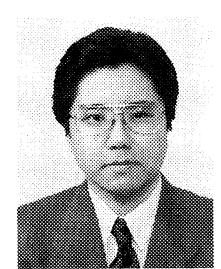

Jun'ya Shimizu received the B.S. and M.S. degrees from Hokkaido University, Sapporo, Japan, in 1992 and 1994, respectively. $\mathrm{He}$ is currently a Ph.D student in the Division of Electronics and Information Engineering, Hokkaido University and a Research Fellow of the Japan Society for the 


\section{J. SHIMIZU et al.: SIGNAL ENHANCEMENT USING ROBUST TLS ALGORITHM}

Promotion of Science. His research interests are in the areas of active sound control, adaptive signal processing, and multirate signal processing. He is a member of ASJ, IEEE and the Institute of Electronics, Information and Communication Engineers.

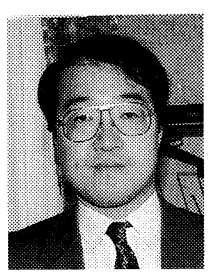

Yoshikazu Miyanaga was born in Sapporo, Japan, on December 20, 1956. He received the B.S., M.S., and Dr.Eng. degrees from Hokkaido University, Sapporo, Japan, in 1979, 1981, and 1986, respectively. He was a Research Associate at the Institute of Applied Electricity, Hokkaido University from 1983 to 1987 , and a lecturer of Electronic Engineering at Faculty of Engineering, Hokkaido University from 1987 to 1988. He is currently an Associate Professor of Electronics and Information Engineering at Graduate School of Engineering, Hokkaido University. His research interests are in the areas of adaptive signal processing and parallel computation. Dr. Miyanaga is a member of ASJ, IEEE, the Institute of Electronics, Information and Communication Engineers and Information Processing Society of Japan.

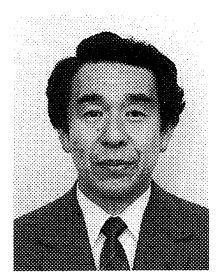

Koji Tochinai was born in Sapporo, Japan, on July 8, 1939. He received the B.S., M.S., and Dr.Eng. degrees from Hokkaido University, Sapporo, Japan, in 1962, 1964 and 1986, respectively. $\mathrm{He}$ is currently a Professor of Electronics and Information Engineering at Graduate School of Engineering and the Director of the Computing Center, Hokkaido University. His research interests are in the areas of document processing and machine translation. Dr. Tochinai is a member of ASJ, the Institute of Electronics, Information and Communication Engineers and Information Processing Society of Japan. 